\title{
Metformin alleviates intervertebral disc degeneration by upregulating MMP-1 expression via the KDM6A/ SOX9/miR-202-3p/MMP-1 signaling pathway
}

\author{
Xin-yao $\mathrm{Hu}^{1}$, Dong-zi $\mathrm{CaO}^{2}$, Zhi-hua Wang ${ }^{3}$
}

\begin{abstract}
${ }^{1}$ Ankang Hospital of Traditional Chinese Medicine, Ankang, Shaanxi Province, China ${ }^{2}$ Yangling Demonstration Zone Hospital, Yangling, Shaanxi Province, China ${ }^{3}$ The Second Affiliated Hospital of Kunming Medical University, Kunming, Yunnan Province, China
\end{abstract}

Submitted: 16 December 2019; Accepted: 21 January 2020 Online publication: 25 March 2021

Arch Med Sci

DOI: https://doi.org/10.5114/aoms/117427

Copyright @ 2021 Termedia \& Banach

\section{Abstract}

Introduction: MiR-202-3p is involved in the pathogenesis of intervertebral disc degeneration (IDD) via regulating MMP-1 expression in neuronal precursor cells (NPCs). As an activator of miR-202-3p and KDM6A expression, metformin (MET) may regulate the expression of $S 0 \times 9$ via regulating the methylation status of the SOX9 promoter. However, the role of MET in the treatment of IDD remains to be explored.

Material and methods: Quantitative real-time PCR was performed to analyze the expression of KDM66, SOX9, MMP-1 and miR-202-3p in NPCs and IDD rabbits. Western blot was carried out to eyaluate the expression of KDM6A, SOX9 and MMP-1 protein under different conditions. Bisulfite sequencing PCR was used to analyze the DNA methylation of the SOX9 promoter. Luciferase assay was carried out to explore the inhibitory effect of miR-202-3p upon MMP-1.

Results: The expression of KDM6A, SOX9 and MMP-1 was abnormal in IDD cells and rabbits, while the MET treatment restored the normal expression of KDM6A, SOX9 and MMP-1 and miR-202-3p. Mechanistically, MET treatment reduced the level of hypermethylation of the SOX9 promoter, thus restoring the expression of SOX9 in IDD cells and rabbits. The elevation in SOX9 expression promoted the expression of miR-202-3p, therefore inhibiting the expression of MMP-1, a downstream target of miR-202-3p.

Conclusions: In this study, we set up cellular and animal models of IDD and treated them with MET to probe the effect of MET on IDD and the signaling pathway of KDM6A/SOX9/miR-202-3p/MMP-1. Our work provided deep insight into the molecular mechanism underlying the therapeutic role of MET in the treatment of IDD.

Key words: intervertebral disc degeneration, nucleus pulposus cells, metformin, KDM6A, SOX9, miR-202-3p, MMP-1.

\section{Introduction}

Degenerative disorders of the spinal cord are significant health issues faced by the global population, and most cases of degenerative disorders of the spinal cord are triggered by intervertebral disc degeneration (IDD) $[1,2]$. While the detailed mechanisms underlying the pathogenesis of IDD remain unclear, major progress has been made to elucidate relevant
Corresponding author: Prof. Zhi-hua Wang The Second Affiliated Hospital of Kunming Medical University, Kunming Yunnan Province, China E-mail: doxspine@yeah.net 
molecular pathways involved in the pathogenesis of IDD. The onset of IDD is a process involving multiple factors, including a critical step involving extracellular matrix degradation. NP plays an important role in maintaining the biomechanical properties of the intervertebral discs in the course of IDD [3]. In addition, as the primary composition in the extracellular matrix, proteoglycans, aggrecan and collagens play a vital role in maintaining the normal functions of the intervertebral discs. In fact, the loss of aggrecan and collagen functions has been correlated with the onset of IDD [4]. Furthermore, the balance between MMPs and TIMPs is possibly involved in the pathogenesis and progression of IDD [5]. For example, Deng et al. found that the expression level of MMP-1 in degenerated discs is significantly increased [6]. In addition, Baillet et al. found that an increased level of MMP-1 expression could be induced by exposing the intervertebral discs to surgical injuries [7]. Xu et al. found that the level of MMP-1 expression is significantly correlated with older age as well as the severity of IDD [8]. Overall, the above results suggested that MMP-1 plays an important role in the onset of IDD.

Past studies demonstrated that short microRNAs, also termed miRNAs, are implicated functionally in the control of many biological and physiological activities, including cell differentiation, cell apoptosis, and cell proliferation [9]. In addition, miRNAs are also implicated in many human disorders, such as the onset of cardiovascular diseases as well as tumorigenesis [10]. By binding to the $3^{\prime}$ untranslated region (3' UTR) of their target genes, miRNAs reduce the expression levels of these genes [11-13]. Nevertheless, few studies have focused on the regulatory relationship between the pathogenesis of IDD and miRNAs [14]. A miRNA named miR-202-3p is suspected to play a key role in the pathogenesis of IDD. For example, the reduced expression of miR-202-3p in the presence of $\mathrm{IL}-1 \beta$ is associated with the increased expression level of MMP-1 in neuronal precursor cells (NPCs). In addition, by inducing the expression of MMP-1, $1 \mathrm{~L}-1 \beta$ can activate MAPK as well as NF- $\kappa B$ signaling to promote the progression of IDD. On the other hand, a reduced expression level of miR-202-3p remarkably increases the expression level of MMP-1. The H3K27me3 family of demethylases contains several key family members, such as KDM7A, KDM6A, and KDM6B, all of which can interact with the tri-methyl groups in their targets to specifically overcome certain factors leading to gene repression during tissue differentiation. As a compound frequently used as a nutritional supplement, metformin (MET) treatment has been reported to stabilize the level of circulating PCSK9 in stable coronary artery disease (SCAD) patients [5]. Also, MET can directly act on the activation of certain key signaling pathways involved in senescence and aging, including the UTX/KDM6A pathway [15]. In addition, MET can suppress the demethylating function of UTX and KDM6A enzymes.

MiR-202-3p is involved in the pathogenesis of IDD via regulating MMP-1 expression in NPCS [16]. Furthermore, as an activator of miR-202-3p and KDM6A, MET may regulate the expression of SOX9 via regulating the methylation status of the SOX9 promoter $[15,17]$. In this study, we set up an animal model of IDD and treated it with MET to probe the effect of MET on the signaling pathway of KDM6A/SOX9/miR-202-3p/MMP-1 and the treatment of IDD.

\section{Material and methods}

Establishment of an animal model of IDD via the noninvasive induction of cumulative axial load

A total of 36 New Zealand male white rabbits (about 5 months of age, weighing 2.2-2.6 kg) were acquired from our laboratory animal center and housed in our animal facility for 7 days of adaptation. Afterwards, the rabbits were assigned randomly to three treatment groups, i.e., 1 - an NC group; 2 - an IDD group; and 3 - an IDD + MET group, with 12 rabbits in each group. During the experiment, all rabbits used to establish the IDD model (i.e., groups 2 and 3) were housed in plastic cages and their posture was maintained in an upright position, and a collar load with a weight of $600 \mathrm{~g}$ was hanging on the neck of each rabbit to provide a load induction, which was carried out for 8 h every day. After 8 weeks of induction, the successful establishment of a rabbit IDD model was confirmed by the procedures described below. In the control group, the rabbits were not treated by load induction. In the IDD + MET group, the IDD rabbits were further treated for these 8 weeks with a dose of $30 \mathrm{mg} / \mathrm{kg} /$ day of MET given orally. During the entire experiment, the temperature in the animal facility was maintained at $22 \pm 3^{\circ} \mathrm{C}$, while the humidity in the animal facility was maintained at $55 \pm 5 \%$. The entire experiment was carried out in compliance with the Guideline for the Care and Use of Laboratory Animals.

\section{Culture of neuronal precursor cells}

Neuronal precursor cells were isolated from NP tissue samples using a method previously described [18]. In brief, all tissue samples were rinsed with PBS, then digested at $37^{\circ} \mathrm{C}$ for 30 min using F12 medium (Gibco, Grand Island, NY) containing $2 \mathrm{U} / \mathrm{ml}$ of protease. After being treated at $37^{\circ} \mathrm{C}$ for $4 \mathrm{~h}$ using $0.25 \mathrm{mg} / \mathrm{ml}$ of type II collagenase (Gibco, Grand Island, NY), the lysate of NP tissue 
samples was prepared into single cell suspensions and then transferred to a $50 \mu \mathrm{m}$ cell strainer (BD Biosciences, Franklin Lakes, NJ) before the lysate was centrifuged for $10 \mathrm{~min}$ at $1000 \mathrm{~g}$. Finally, the isolated NPCs were suspended in a DMEM medium with $1 \%$ L-glutamine, $100 \mu \mathrm{g} / \mathrm{ml}$ streptomycin, $100 \mathrm{U} / \mathrm{ml}$ penicillin, as well as 10\% FBS (Gibco, Grand Island, NY), and cultured for 1 week under $5 \% \mathrm{CO}_{2}$ and $37^{\circ} \mathrm{C}$ before they were collected for subsequent analyses.

\section{Stimulation of neuronal precursor cells}

To stimulate NPCs, they were starved for $12 \mathrm{~h}$ in a medium containing no FBS and then exposed to $6 \mathrm{~h}$ of IL-1 $\beta$ ( $5 \mathrm{ng} / \mathrm{ml}$, Peprotech, Rocky Hill, NJ) treatment to stimulate the cells [19].

\section{Bisulfite sequencing}

Bisulfite real-time PCR sequencing was carried out to determine the level of DNA methylation in the promoter of the SOX9 gene in collected tissue and cell samples. In brief, the samples were first immersed in a buffer containing $0.1 \mathrm{mM}$ of EDTA and $10 \mathrm{mM}$ of Tris, and then treated with a Bioruptor sonicator to fragment the DNA in the samples. In the next step, the fragmented DNA was precipitated using ethanol, treated using an End-It DNA Repair assay kit (Illumina, Hayward, CA) in accordance with the instructions of the manufacturer to repair the end of the DNA sample, and re-purified using a MinElute DNA clean up assay kit (Qiagen, Hilden Germany) in accordance with the instructions of the manufacturer before carrying out the ligation reaction using a Quick Ligation assay kit (NEB, Ipswich, MA) in accordance with the instructions of the manufacturer. The ligated DNA was then separated using a $5 \%$ agarose gel (Bio-Rad, Hercules, CA) and then treated using a MethylEasy assay kit (Bio-Rad, Hercules, CA) in accordance with the instructions of the manufacturer. Finally, the DNA methylation status in the promoter of the SOX9 gene was determined via real-time PCR using a Platinum Taq buffer (Invitrogen, Carlsbad, CA) containing $1 \mathrm{U}$ of Platinum Taq reagent, $2.5 \mathrm{mM}$ of $\mathrm{MgCl}_{2}$ and $50 \mu \mathrm{M}$ of dNTPs. The PCR conditions were set up in accordance with the instructions of the kit manufacturer.

\section{RNA isolation and real-time PCR}

Real-time PCR was carried out to measure the mRNA expression of KDM6A, SOX9, MMP-1, and miR-202-3p in various samples. In brief, total RNA content in each sample was extracted using a Trizol reagent (Invitrogen, Carlsbad, CA) in accordance with the instructions of the kit manufacturer. Then, extracted total RNA was converted to cDNA by reverse transcription, and the mRNA expression of KDM6A (forward primer: 5'-AGCGCAAAGGAGCCGTGGAAAA-3'; reverse primer: 5'-GTCGTTCACCATTAGGACCTGC-3'), SOX9 (forward primer: 5'-AGGAAGCTCGCGGACCAGTAC-3'; reverse primer: 5'-GGTGGTCCTTCTTGTGCTGCAC-3'), MMP-1 (forward primer: 5'-ATGAAGCAGCCCAGATGTGGAG-3'; reverse primer: 5'-TGGTCCACATCTGCTCTTGGCA-3'), and miR-202-3p (forward primer: 5'-TTCCTATGCATATACTTCTT-3'; reverse primer: 5'-GAACATGTCTGCGTATCTC-3') in each sample was measured using TaqMan real-time PCR (Applied Biosystems, Foster City, CA).

\section{Cell culture, treatment and transfection}

The NPCs culture was carried out under 5\% $\mathrm{CO}_{2}$, saturated humidity and $37^{\circ} \mathrm{C}$. When the cells were confluent, they were randomly divided into 3 groups, i.e., 1 . untreated group; 2 . IL-1 $\beta$ group $(5 \mathrm{ng} / \mathrm{ml})$; and 3. IL-1 $\beta(5 \mathrm{ng} / \mathrm{ml})+$ MET group $(1 \mu \mathrm{M})$. The cells in various groups were treated with designated concentrations of $\mathrm{HL}-1 \beta$ and MET or PBS for $12 \mathrm{~h}$ before they were collected for subsequent assays. In another set of experiments, NPCs were randomly divided into another 3 groups, i.e., 1 - a scramble control group; 2 - a miR-202-3p precursor group; and 3 - an MMP-1 siRNA group. The cells in various groups were treated with different nucleotides for $48 \mathrm{~h}$ using Lipofectamine 3000 (Invitrogen, Carlsbad, CA) in accordance with the instructions of the kit manufacturer before the cells were collected and the expression of target genes in the cells was measured.

\section{Vector construction, mutagenesis and luciferase assay}

The results of our previous sequence analysis indicated that miR-202-3p could bind to the 3' UTR of MMP-1. In order to further explore the regulatory role of miR-202-3p in MMP-1 expression, a pcDNA luciferase plasmid (Promega, Madison, WI) containing the wild type 3' UTR of MMP-1 was established. At the same time, site-directed mutagenesis was carried out in the miR-202-3p binding site located in the 3' UTR of MMP-1 using a Quick Change site directed mutagenesis assay kit (Stratagene, San Diego, CA) in accordance with the instructions of the kit manufacturer to generate the mutant type sequence of the $3^{\prime}$ UTR of MMP-1, which was also inserted into a pcDNA luciferase plasmid to establish the luciferase plasmid containing the mutant type $3^{\prime}$ UTR of MMP-1. In the next step, both the mutant type and wild type 3' UTR of MMP-1 were co-transfected into NPCs in conjunction with either a miR-202-3p mimic or a miR-202-3p inhibitor. The luciferase activity of transfected cells was measured $48 \mathrm{~h}$ later using a Bright Glo luciferase assay kit (Promega, Madi- 
son, $\mathrm{WI}$ ) in accordance with the instructions of the kit manufacturer.

\section{Western blot analysis}

Western blot was carried out to measure the protein expression of KDM6A, SOX9, and MMP-1. In brief, tissue and cell samples were exposed to primary anti-KDM6A, anti-SOX9, and anti-MMP-1 antibodies ( $1: 100$ dilution, Abcam, Cambridge, MA) at 4 degrees overnight and then incubated for $2 \mathrm{~h}$ at $37^{\circ} \mathrm{C}$ with horseradish peroxidase conjugated secondary IgG antibodies (Cell Signaling Technology, Danvers, MA) before the samples were visualized using a Clarity Western ECL Substrate (Bio-Rad, Hercules, $C A$ ) to determine the protein expression of KDM6A, SOX9, and MMP-1 in each sample.

\section{Statistical analysis}

All experimental data were analyzed using Version 5 Expert StatView statistical software (SAS,

A


Cary, NC). The data were analyzed by t-test and one-way analysis of variance (ANOVA). The results were presented as mean \pm standard deviation. The level of significance was set to 0.05 .

\section{Results}

\section{MET treatment alleviated deregulation of KDM6A, SOX9, MMP-1 and miR-202-3p in NPCS}

NPCs were treated with IL-1 $\beta$ alone or in combination with MET before Western blot was performed to analyze the differential expression of KDM6A, SOX9 and MMP-1. IL-1 $\beta$ treatment remarkably repressed the expression of KDM6A (Figures $1 \mathrm{~A}, \mathrm{~B}$ ) and SOX9 (Figure $1 \mathrm{~A}, \mathrm{C}$ ), while the treatment with MET restored the normal expression of KDM6A and SOX9 to a certain level. In contrast, the expression of MMP-1 was dramatically enhanced by IL-1 $\beta$ while the MET treatment reduced the expression of MMP-1 in NPCs exposed to IL-1 $\beta$ (Figure $1 \mathrm{~A}, \mathrm{D}$ ).
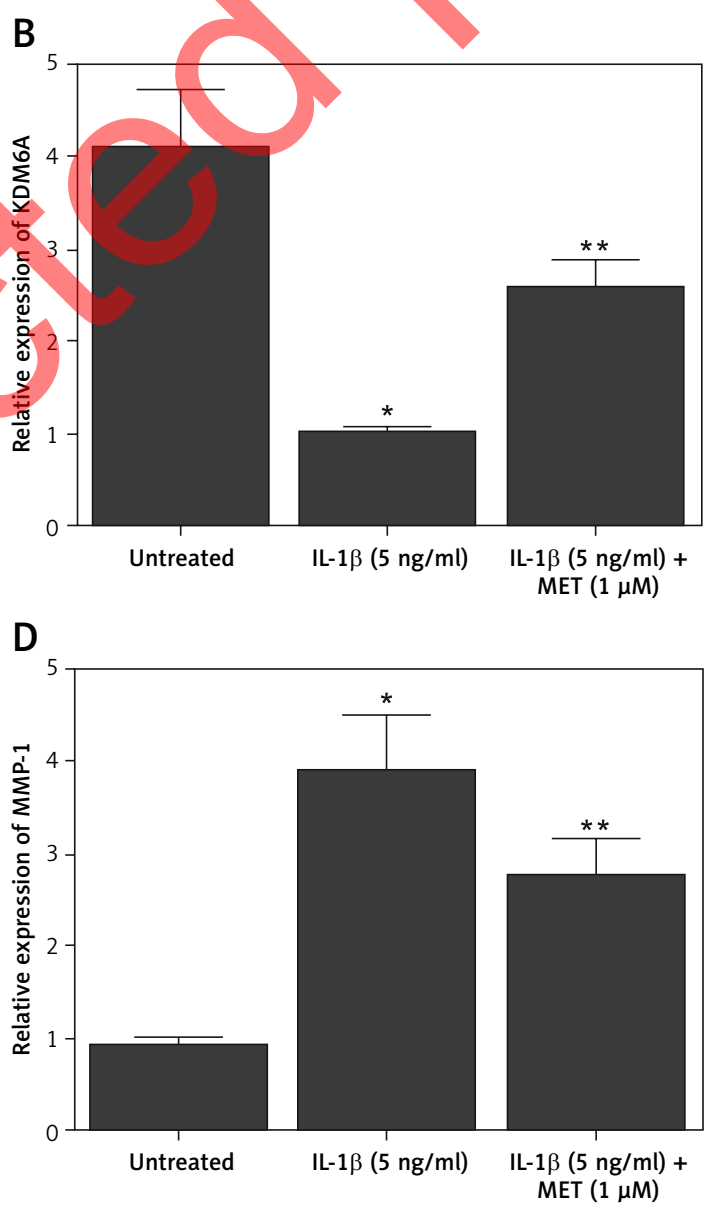

Figure 1. IL-1 $\beta$ induced deregulation of KDM6A, SOX9 and MMP-1 protein expression, and MET treatment reversed the effect of IL-1 $\beta\left({ }^{*} p<0.05\right.$, vs. untreated group; ${ }^{* *} p<0.05$, vs. IL-1 $\beta$ group). A - Western blot analysis showed that IL-1 $\beta$ inhibited the expression of KDM6A and SOX9 while activating the expression of MMP-1 protein in NPCs, and MET treatment reversed the effect of IL-1 $\beta$. B - Quantitative analysis showed that IL- $1 \beta$ inhibited expression of KDM6A protein in NPCs and MET treatment reversed the effect of IL-1B.C - Quantitative analysis showed that IL-1 $\beta$ inhibited expression of SOX9 protein in NPCs and MET treatment reversed the effect of IL-1 $\beta$. D - Quantitative analysis showed that IL-1 $\beta$ activated expression of MMP-1 protein in NPCs and MET treatment reversed the effect of IL-1 $\beta$ 
Additionally, quantitative real-time PCR was performed to evaluate the mRNA expression of KDM6A, SOX9, MMP-1, and miR-202-3p in NPCs placed under different conditions. The expression of KDM6A (Figure 2 A), SOX9 (Figure 2 B) and MMP-1 (Figure 2 D) mRNA was notably repressed by IL-1 $\beta$ treatment, while the treatment with MET effectively restored the normal expression of KDM6A, SOX9 and MMP-1 mRNA in IL-1 $\beta$-treated NPCs. However, the expression of miR-202-3p was apparently elevated by IL-1 $\beta$ treatment, while the treatment with MET reduced miR-202-3p expression in NPCs exposed to IL-1 $\beta$ (Figure $2 \mathrm{C}$ ).

\section{MET treatment reduced hypermethylation of the SOX9 promoter in NPCS}

DNA methylation plays an important role in gene regulation. In this study, we performed bisulfite sequencing PCR to analyze the different levels of DNA methylation of the SOX9 promoter in NPCs exposed to different conditions. IL-1 $\beta$ treatment significantly increased DNA methylation of

A


the SOX9 promoter and down-regulated SOX9 expression. MET treatment notably suppressed hypermethylation of the SOX9 promoter in NPCS (Figure 3).

\section{MiR-202-3p repressed expression of MMP-1 through binding to its 3' UTR}

Sequence analysis indicated that miR-202-3p could bind to the $3^{\prime}$ UTR of MMP-1. In order to further explore the regulatory role of miR-202$3 p$ in MMP-1 expression, a luciferase plasmid containing the MMP-1 $3^{\prime}$ UTR was established and co-transfected into NPCs in conjunction with either a miR-202-3p mimic or a miR-202-3p inhibitor. The luciferase activity of MMP-1 was significantly repressed by the miR-202-3p mimic and enhanced by the miR-202-3p inhibitor (Figure 4 A). Quantitative real-time PCR and Western blot were performed to evaluate the expression of MMP-1 mRNA and protein in NPCs transfected with a miR-202-3p precursor and MMP-1 siRNA, respectively. The expression of MMP-1 mRNA and


Figure 2. IL-1 $\beta$ induced deregulation of KDM6A, SOX9, miR-202-3p and MMP-1 mRNA expression while MET treatment reversed the effect of IL- $1 \beta$ ( ${ }^{*} p<0.05$, vs. untreated group; ${ }^{* \star} p<0.05$, vs. IL- $1 \beta$ group). A - IL- $1 \beta$ repressed KDM6A mRNA expression in NPCs and MET treatment reversed the effect of IL-1 $\beta$. B - IL-1 $\beta$ repressed SOX9 mRNA expression in NPCs and MET treatment reversed the effect of IL-1 3 . C - IL-1 $\beta$ activated miR-202-3p expression in NPCs and MET treatment reversed the effect of IL-1 $\beta$. D - IL-1 $\beta$ repressed MMP- 1 mRNA expression in NPCs and MET treatment reversed the effect of IL-1 $\beta$ 
Untreated

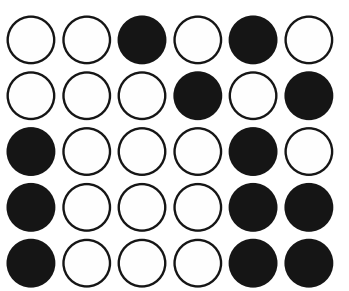

$\mathrm{IL}-1 \beta(5 \mathrm{ng} / \mathrm{ml})$

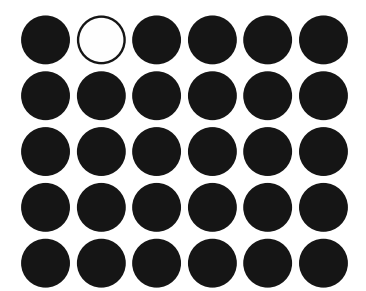

$\mathrm{IL}-1 \beta(5 \mathrm{ng} / \mathrm{ml})+\mathrm{MET}(1 \mu \mathrm{M})$



Figure 3. IL-1 $\beta$ induced hypermethylation of SOX9 promoter and MET treatment reversed the effect of IL-1 $\beta$ in NPCS

A

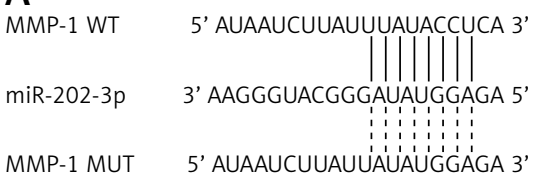

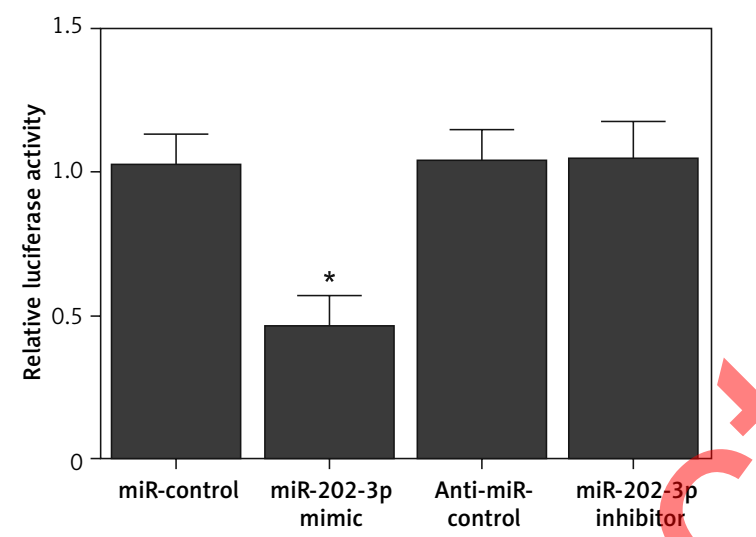

B

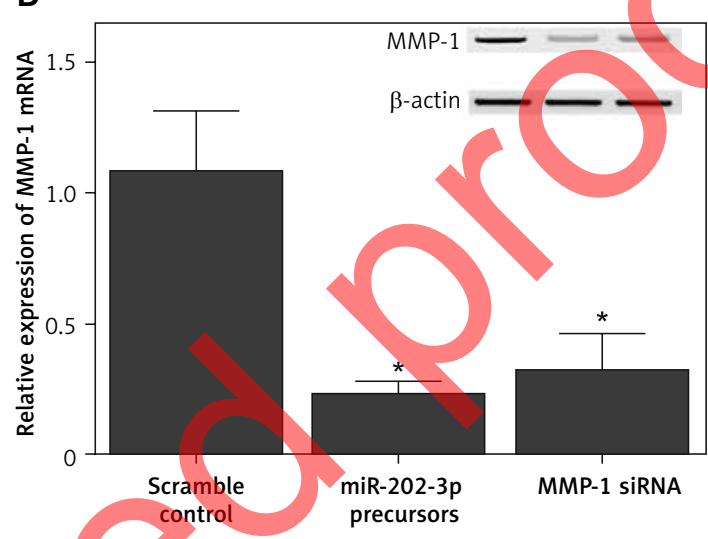

Figure 4. MiR-202-3p inhibited the expression of MMP-1 through binding to its $3^{\prime}$ UTR. A - The luciferase activity of MMP-1 vector was inhibited by miR-202-3p mimics and increased by miR-202-3p inhibitors $\left({ }^{*} p<0.05\right.$, vs. miR-controls). B - The expression of MMP-1 protein was effectively inhibited by miR-202-3p precursors and MMP-1 siRNA $\left({ }^{*} p<0.05\right.$, vs. scramble controls)

protein was notably inhibited by both the miR202-3p precursor and MMP-1 siRNA (Figure 4 B).

MET administration restored the abnormal expression of KDM6, SOX9, MMP-1 and miR-202-3p in IDD rabbits

An IDD (intervertebral disc degeneration) rabbit model was established and the value of disc height index (DHI) was measured at different time points. In the control rabbits, the $\mathrm{DHI}$ was progressively decreased. However, the DHI for IDD rabbits remained stable at different time points, indicating the abnormal elevation of DHI when compared with the control. MET administration could restore the normal value of $\mathrm{DHI}$ in IDD rabbits to a certain degree (Figure $5 \mathrm{~A}$ ). Additionally, we performed Western blot to evaluate the expression of KDM6, SOX9 and MMP-1 protein in IDD rabbits. The abnormally up-regulated KDM6 (Figure 5 B) and SOX9 (Figure $5 \mathrm{C}$ ) expression as well as the abnormally down-regulated MMP-1 (Figure 5 D) expression was restored to normal by MET treat- ment. Moreover, the $\mathrm{qPCR}$ results showed that MET treatment decreased the expression of KDM6A (Figure $6 \mathrm{~A}$ ), SOX9 (Figure $6 \mathrm{~B}$ ) and MMP-1 (Figure $6 \mathrm{D}$ ) $\mathrm{mRNA}$, while increasing the expression of miR-202-3p (Figure $6 \mathrm{C}$ ) in IDD rabbits. Similar to the change of SOX9 expression in IDD rabbits, the DNA methylation of the SOX9 promoter was significantly increased in IDD rabbits, while MET administration efficiently reduced the hypermethylation of the SOX9 promoter in IDD rabbits (Figure 7).

In conclusion, our results revealed that MET treatment up-regulated KDM6A expression, which then promoted the demethylation of the SOX9 promoter to up-regulate its expression and induce the expression of miR-202-3p. Increased expression of miR-202-3p inhibited the expression of MMP-1 and attenuated the severity of IDD (Figure 8).

\section{Discussion}

As a process involving both senescence and apoptosis, IDD can be alleviated by MET via stimu- 
A

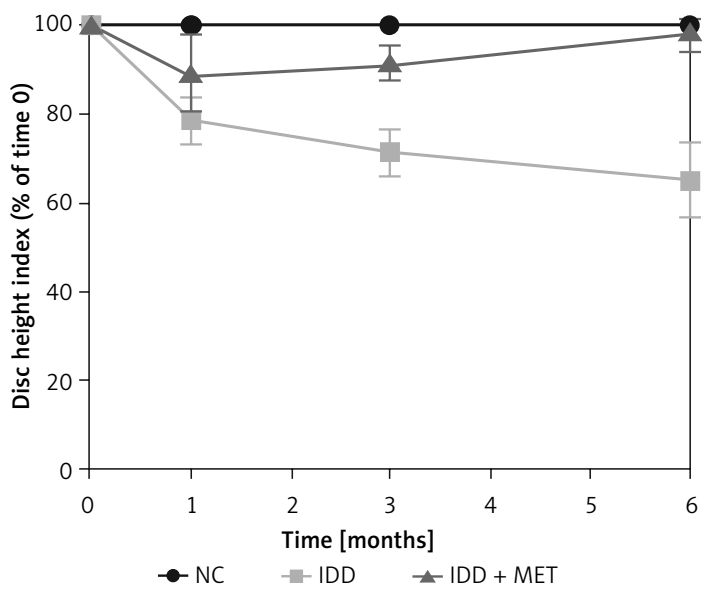

C


lating the activity of autophagy. MET may exert its effects on inhibiting the demethylating activity of H3 Lys27 enzymes by interacting with the protein residues residing in the catalytic domain of UTX and KDM6A. In this study, we treated NPCs with IL-1 $\beta$ to establish a cellular model of IDD. We also constructed an IDD rabbit model to explore the therapeutic efficiency of MET in IDD treatment. Furthermore, we carried out bisulfite sequencing PCR to evaluate the level of DNA methylation of the SOX9 promoter in the IDD models. MET treatment could effectively reduce the level of SOX9

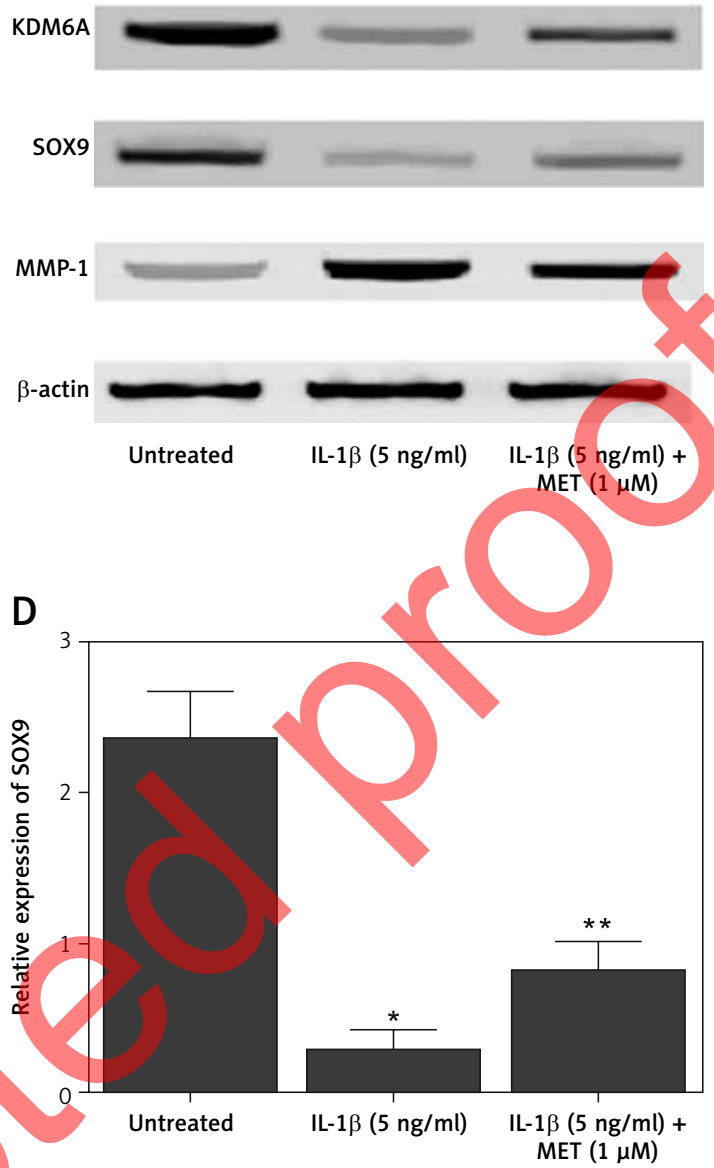

Figure 5. MET treatment effectively restored the deregulation of KDM6A, SOX9 and MMP-1 protein expression in IDD rabbits $\left({ }^{*} p<0.05\right.$, vs. untreated group; ${ }^{* \star} p<0.05$, vs. IL-1 $\beta$ group). A - Graphic representation of $\mathrm{DHI}$ of rabbit discs. $\mathbf{B}$ - Western blot analysis showed that the inhibited expression of KDM6A and SOX9, and activated expression of MMP-1 protein, in IDD rabbits were recovered by MET treatment. C - Quantitative analysis showed that the inhibited expression of KDM6A protein in IDD rabbits was recovered by MET treatment. D - Quantitative analysis showed that the inhibited expression of SOX9 protein in IDD rabbits was recovered by MET treatment. E - Quantitative analysis showed that the activated expression of MMP-1 protein in IDD rabbits was recovered by MET treatment

promoter methylation in NPCs and IDD rabbits stimulated by IL-1 $\beta$.

KDM6A, also termed lysine specific demethylase 6A and UTX, acts as a demethylase specific to histone H3K27. In fact, KDM6A plays a critical role in regulating the differentiation of stem cells toward M2 macrophages [20]. In addition, the over-expression of KDM6A in MSCs can promote osteogenesis while inhibiting adipogenesis [21]. In fact, the methylation of histone lysine is under the control of histone lysine demethylase (KDM) and histone lysine methyltransferase (KMT). 
A

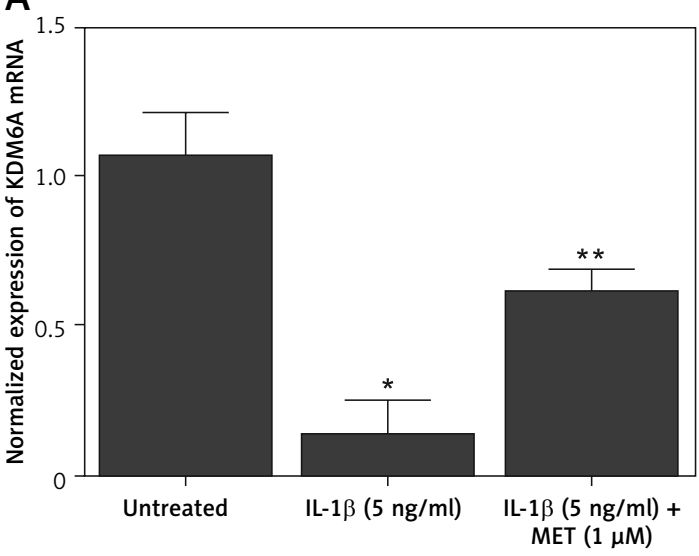

C

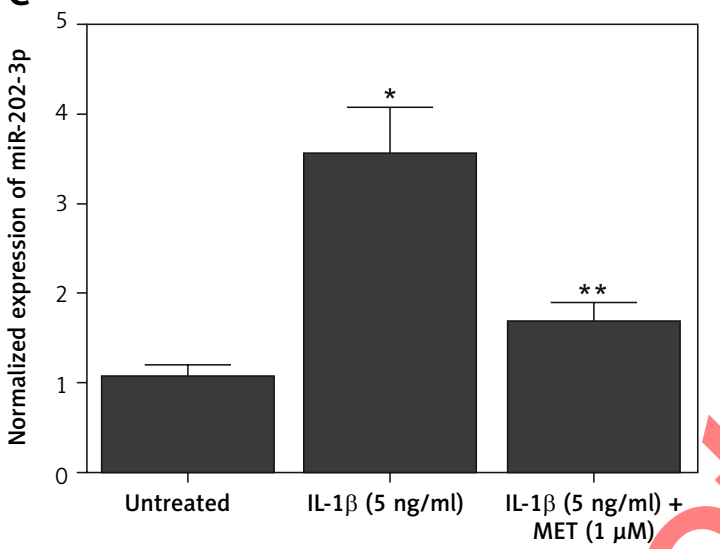

B



D

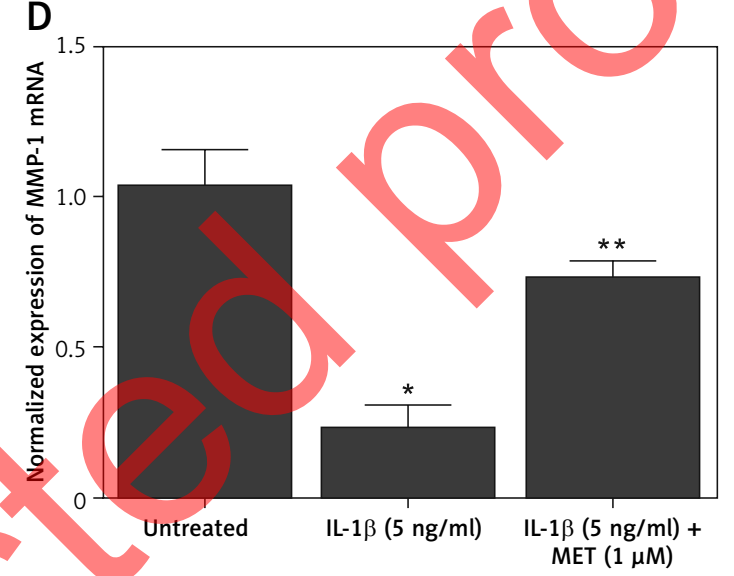

Figure 6. The deregulated mRNA expression of KDM6A, SOX9, MMP-1 and miR-202-3p in IDD rabbits was recovered by MET treatment ( ${ }^{*} p<0.05$, vs. untreated group; ${ }^{* *} p<0.05$, vs. IL-1 $\beta$ group). A - Repressed expression of KDM6A mRNA in IDD rabbits was restored by MET treatment. B - Repressed expression of SOX9 mRNA in IDD rabbits was restored by MET treatment. C - Enhanced expression of miR-202-3p in IDD rabbits was restored by MET treatment. $\mathbf{D}$ - Repressed expression of MMP-1 MRNA in IDD rabbits was restored by MET treatment
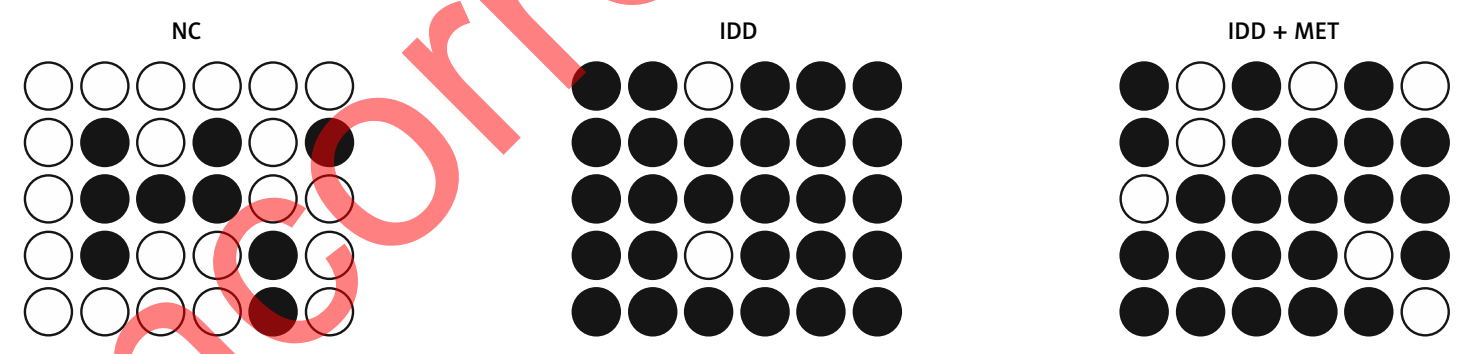

Figure 7. Hypermethylated SOX9 promoter in IDD rabbits was demethylated by MET treatment

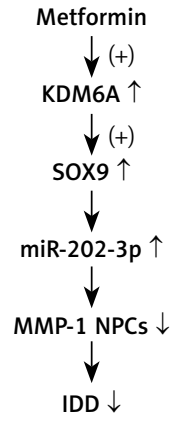

Figure 8. Diagram showing the regulatory role of MET treatment in IDD
SOX9 is known as an important transcription factor involved in chondrogenic differentiation. Past results demonstrated that the level of SOX9 protein expression is apparently reduced after the expression of KDM6A is knocked down in PDLSC cells. In fact, as a key regulator involved in the differentiation of Sertoli cells, SOX9 can affect the expression of a wide range of target genes in both human and mouse [22-25]. In addition, SOX9 was shown previously to control the expression level of pri-miR-140 involved in activation of the Wwp2 gene $[26,27]$. 
Growing evidence suggests that a wide range of miRNAs are involved in the onset of IDD by regulating the extent of nucleus pulposus cell apoptosis as well as degradation of the extracellular matrix. In addition, the expression level of miR-30d is negatively associated with the expression level of SOX9 in NP tissues. On the other hand, the expression level of miR-202-5p/3p is low in mice with the Sox9 gene knocked out, indicating that pri-miR-202 acts as a downstream target of SOX9. In this study, we performed luciferase assays to explore the inhibitory role of miR-202-3p in MMP-1 expression. The luciferase activity of the MMP-1 vector was significantly repressed by miR-202-3p mimics and increased by miR-202-3p inhibitors. As a regulator in the expression of MMP-1, miR-202$3 p$ expression can suppress the expression level of MMP-1 in the presence of IL-1 $\beta$. On the other hand, transfection using miR-202-3p inhibitors apparently promoted the production of MMP-1, suggesting that miR-202-3p can reduce the expression level of MMP-1 in NP cells to participate in the onset of IDD.

As enzymes participating in the extracellular matrix degradation, MMPs, especially MMP-1, can cleave endogenous type I, type II, as well as type III collagens in the interstitial space, leading to possible disc degeneration and alterations in the disc structure [18, 28-30]. In addition, the expression levels of MMP-3 and MMP-1 are both increased during the onset of lumbar disc degeneration [31]. In a similar study, it was found that the level of MMP-1 expression was significantly associated with age [8].

In summary, the findings of this study demonstrated that miR-202-3p is involved in the pathogenesis of IDD via regulating MMP-1 expression in NPCs. Furthermore, as an activator of miR-202-3p and KDM6A, MET may regulate the expression of SOX9 via regulating the methylation status of the SOX9 promoter. Meanwhile, treatment with MET may enhance KDM6A expression. In this study, we set up an animal model of IDD and treated it with MET to probe the effect of MET on the signaling pathway of KDM6A/SOX9/miR-202-3p/MMP-1 and the treatment of IDD.

\section{Conflict of interest}

The authors declare no conflict of interest.

\section{References}

1. Andersson GB. Epidemiological features of chronic lowback pain. Lancet 1999; 354: 581-5.

2. Waddell G. Low back pain: a twentieth century health care enigma. Spine (Phila Pa 1976) 1996; 21: 2820-5.

3. Ma K, Chen S, Li Z, et al. Mechanisms of endogenous repair failure during intervertebral disc degeneration. Osteoarthritis Cartilage 2019; 27: 41-8.
4. Kepler CK, Ponnappan RK, Tannoury CA, Risbud MV, Anderson DG. The molecular basis of intervertebral disc degeneration. Spine J 2013; 13: 318-30.

5. Shek AB, Alieva RB, Kurbanov RD, Hoshimov SU, Nizamov UI, Ziyaeva AV. Can metformin stabilize PCSK9 level in stable coronary artery disease patients treated with statins? Arch Med Sci Atheroscler Dis 2019; 4: e144-50.

6. Deng B, Ren JZ, Meng XQ, et al. Expression profiles of MMP-1 and TIMP-1 in lumbar intervertebral disc degeneration. Genet Mol Res 2015; 14: 19080-6.

7. Baillet A, Grange L, Trocme C, et al. Differences in MMPS and TIMP-1 expression between intervertebral disc and disc herniation. Joint Bone Spine 2013; 80: 341-2.

8. Xu H, Mei Q, He J, Liu G, Zhao J, Xu B. Correlation of matrix metalloproteinases- 1 and tissue inhibitor of metal loproteinases-1 with patient age and grade of lumbar disk herniation. Cell Biochem Biophys 2014; 69: 439-44.

9. Kim J, Kang Y, Kojima Y, et al. An endothelial apelin-FGF link mediated by miR-424 and miR-503 is disrupted in pulmonary arterial hypertension. Nat Med 2013; 19: 74-82.

10. Vaira V, Faversani A, Dohi T, et al. miR-296 regulation of a cell polarity-cell plasticity module controls tumor progression. Oncogene 2012; 31: 27-38.

11. Catuogno S, Cerchia L, Romano G, Pognonec P, Condorelli $G$, de Franciscis V. miR-34c may protect lung cancer cells from paclitaxel-induced apoptosis. Oncogene 2013; 32: 341-51

12. Chuang IC, Jones PA. Epigenetics and microRNAs. Pediatr Res 2007; 61: 24R-9R.

3. Jansson MD, Lund AH. MicroRNA and cancer. Mol Oncol 2012; 6: 590-610.

14. Yu X, Li Z, Shen J, et al. MicroRNA-10b promotes nucleus pulposus cell proliferation through RhoC-Akt pathway by targeting HOXD10 in intervetebral disc degeneration. PLoS One 2013; 8: e83080.

15. Cuyas E, Verdura S, Llorach-Pares L, et al. Metformin directly targets the H3K27me3 demethylase KDM6A/UTX. Aging Cell 2018; 17: e12772.

16. Shi C, Wu L, Lin W, et al. MiR-202-3p regulates interleukin-1beta-induced expression of matrix metalloproteinase 1 in human nucleus pulposus. Gene 2019; 687: 156-65.

17. Wainwright EN, Jorgensen JS, Kim Y, et al. SOX9 regulates microRNA miR-202-5p/3p expression during mouse testis differentiation. Biol Reprod 2013; 89: 34.

18. Hu B, Shi C, Xu C, et al. Heme oxygenase-1 attenuates IL-1beta induced alteration of anabolic and catabolic activities in intervertebral disc degeneration. Sci Rep 2016; 6: 21190.

19. Shin-Young P, Min-Jeong K, Joong-Soo H. Interleukin-1 beta promotes neuronal differentiation through the Wnt5a/RhoA/JNK pathway in cortical neural precursor cells. Mol Brain 2018; 11: 39.

20. Van der Meulen J, Speleman F, Van Vlierberghe P. The H3K27me3 demethylase UTX in normal development and disease. Epigenetics 2014; 9: 658-68.

21. Hemming S, Cakouros D, Isenmann S, et al. EZH2 and KDM6A act as an epigenetic switch to regulate mesenchymal stem cell lineage specification. Stem Cells 2014; 32: 802-15.

22. Wagner T, Wirth J, Meyer J, et al. Autosomal sex reversal and campomelic dysplasia are caused by mutations in and around the SRY-related gene SOX9. Cell 1994; 79: 1111-20.

23. Chaboissier MC, Kobayashi A, Vidal VI, et al. Functional analysis of Sox8 and Sox9 during sex determination in the mouse. Development 2004; 131: 1891-901. 
24. Vidal VP, Chaboissier MC, de Rooij DG, Schedl A. SOX9 induces testis development in XX transgenic mice. Nat Genet 2001; 28: 216-7.

25. Arango NA, Lovell-Badge R, Behringer RR. Targeted mutagenesis of the endogenous mouse Mis gene promoter: in vivo definition of genetic pathways of vertebrate sexual development. Cell 1999; 99: 409-19.

26. Nakamura Y, Yamamoto K, He X, et al. Wwp2 is essential for palatogenesis mediated by the interaction between Sox 9 and mediator subunit 25. Nat Commun 2011; 2: 251.

27. Yamashita S, Miyaki S, Kato Y, et al. L-Sox5 and Sox6 proteins enhance chondrogenic miR-140 microRNA expression by strengthening dimeric Sox9 activity. J Biol Chem 2012; 287: 22206-15.

28. Vo NV, Hartman RA, Yurube T, Jacobs LJ, Sowa GA, Kang JD. Expression and regulation of metalloproteinases and their inhibitors in intervertebral disc aging and degeneration. Spine J 2013; 13: 331-41.

29. Vincenti MP, Brinckerhoff CE. Transcriptional regulation of collagenase (MMP-1, MMP-13) genes in arthritis: integration of complex signaling pathways for the recruitment of gene-specific transcription factors. Arthritis Res 2002; 4: 157-64.

30. Basaran R, Senol M, Ozkanli S, Efendioglu M, Kaner T. Correlation of matrix metalloproteinase (MMP) $-1,-2,-3$, and -9 expressions with demographic and radiological features in primary lumbar intervertebral disc disease. J Clin Neurosci 2017; 41: 46-9.

31. Zigouris A, Batistatou A, Alexiou GA, et al. Correlation of matrix metalloproteinases- 1 and -3 with patient age and grade of lumbar disc herniation. J Neurosurg Spine 2011; 14: 268-72. 